\title{
Naloxone dosing in the era of ultra-potent opioid overdoses: a systematic review
}

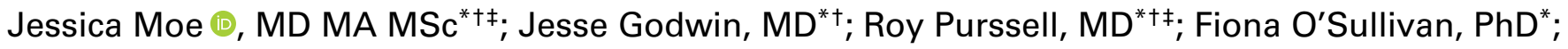 \\ Jeffrey P. Hau, MSc*; Elizabeth Purssell, MD, MSc*; Jason Curran, MPH; ; Mary M. Doyle-Waters, \\ MA, MLISף; Penelope M.A. Brasher, PhDף; Jane A. Buxton, MBBS, MHSc ${ }^{\ddagger \# ; ~ C o r i n n e ~ M . ~ H o h l, ~ M D, ~}$ \\ $\mathrm{MHSc}^{*+\top}$
}

\begin{abstract}
Clinician's Capsule
What is known about the topic?

Fentanyl and ultra-potent opioid prevalence are increasing in North America; however, optimal naloxone dosing for toxicity reversal remains unknown.

What did this study ask?

What is the relationship between naloxone dose (initial and cumulative) and toxicity reversal and adverse events in suspected fentanyl and ultra-potent opioid toxicity?

What did this study find?

Higher initial and cumulative naloxone doses have been used and may be necessary for adequate toxicity reversal. Why does this study matter to clinicians?

Naloxone titration to response may be needed; however, association of dose with adverse events remains unanswered in this population.
\end{abstract}

\section{ABSTRACT}

Objectives: Evaluate the relationship between naloxone dose (initial and cumulative) and opioid toxicity reversal and adverse events in undifferentiated and presumed fentanyl/ ultra-potent opioid overdoses.

Methods: We searched Embase, MEDLINE, Cochrane Central Register of Controlled Trials, DARE, CINAHL, Science Citation Index, reference lists, toxicology websites, and conference proceedings (1972 to 2018). We included interventional, observational, and case studies/series reporting on naloxone dose and opioid toxicity reversal or adverse events in people $>12$ years old.

Results: A total of 174 studies (110 case reports/series, 57 observational, 7 interventional) with 26,660 subjects (median age 35 years; $74 \%$ male). Heterogeneity precluded meta- analysis. Where reported, we abstracted naloxone dose and proportion of patients with toxicity reversal. Among patients with presumed exposure to fentanyl/ultra-potent opioids, $56.9 \%(617 / 1,085)$ responded to an initial naloxone dose $\leq 0.4$ mg compared with $80.2 \%(170 / 212)$ of heroin users, and $30.4 \%(7 / 23)$ responded to an initial naloxone dose $>0.4 \mathrm{mg}$ compared with $59.1 \%(1,434 / 2,428)$ of heroin users. Among patients who responded, median cumulative naloxone doses were higher for presumed fentanyl/ultra-potent opioids than heroin overdoses in North America, both before 2015 (fentanyl/ultra-potent opioids: $1.8 \mathrm{mg}$ [interquartile interval $\{|\mathrm{Q}|\}$, 1.0, 4.0]; heroin: $0.8 \mathrm{mg}$ [IQI, 0.4, 0.8]) and after 2015 (fentanyl/ ultra-potent opioids: $3.4 \mathrm{mg}$ [IQI, 3.0, 4.1]); heroin: $2 \mathrm{mg}$ [IQI, $1.4,2.0])$. Where adverse events were reported, $11 \%$ (490/ 4,414 ) of subjects experienced withdrawal. Variable reporting, heterogeneity and poor-quality studies limit conclusions.

Conclusions: Practitioners have used higher initial doses, and in some cases higher cumulative naloxone doses to reverse toxicity due to presumed fentanyl/ultra-potent opioid exposure compared with other opioids. High-quality comparative naloxone dosing studies assessing effectiveness and safety are needed.

\section{RÉSUMÉ}

Objectif: L'étude visait à évaluer la relation entre les doses de naloxone (initiales et cumulatives) et la neutralisation de la toxicité des opioïdes et les événements indésirables survenus dans les cas tous confondus et dans les cas présumés de surdoses de fentanyl ou d'opioïdes extrêmement puissants.

Méthode: Une recherche documentaire a été entreprise dans les bases de données Embase, MEDLINE, Cochrane Central Register of Controlled Trials, DARE, CINAHL et Science Citation Index ainsi que dans les listes de références bibliographiques, les sites web sur la toxicologie et les actes de congrès

From the *Department of Emergency Medicine, University of British Columbia, Vancouver, BC, Canada; ${ }^{\dagger}$ Department of Emergency Medicine, Vancouver General Hospital, Vancouver, BC, Canada; ${ }^{\ddagger}$ British Columbia Centre for Disease Control, Vancouver, BC, Canada; ${ }^{\S}$ British Columbia Interior Health Authority, Kelowna, BC, Canada; ${ }^{\mathbb{T}}$ Centre for Clinical Epidemiology and Evaluation, Vancouver Coastal Health Research Institute, Vancouver, BC, Canada; and the "School of Population and Public Health, University of British Columbia, Vancouver, BC.

Correspondence to: Dr. Jessica Moe, Department of Emergency Medicine, Vancouver General Hospital, 920 West 10th Avenue, Vancouver, British Columbia, Canada, V5Z 1M9; Email: jessica.moe@ubc.ca

(c) Canadian Association of Emergency Physicians 
(1972 à 2018). Ont été retenues des études d'intervention, des études d'observation et des études ou des séries de cas faisant état de doses de naloxone, de la neutralisation de la toxicité des opioïdes ou d'événements indésirables chez des personnes âgées de plus de 12 ans.

Résultats: Ont été retenues 174 études (110 études ou séries de cas, 57 études d'observation et 7 études d'intervention), totalisant 26660 sujets (âge médian : 35; hommes : 74\%). II n'a pas été possible de procéder à une méta-analyse en raison de l'hétérogénéité des études. Lorsque les données étaient fournies, les chercheurs ont préparé un résumé des doses de naloxone et de la proportion de patients chez qui il y a eu neutralisation de la toxicité. Dans les cas présumés d'exposition au fentanyl ou à des opioïdes extrêmement puissants, 56,9\%(617/1085) des sujets ont réagi à une dose initiale de naloxone $\leq 0,4 \mathrm{mg}$ comparativement à $80,2 \%(170 / 212)$ des utilisateurs d'héroïne, et $30,4 \%(7 / 23)$ ont réagi à une dose initiale de naloxone $>0,4$ mg comparativement à $59,1 \%(1434 / 2428)$ des utilisateurs d'héroïne. Parmi les patients qui ont réagi à la naloxone, les doses médianes cumulatives de naloxone étaient plus élevées dans les cas présumés de consommation de fentanyl ou d'opioïdes extrêmement puissants que dans les cas de surdose d'héroïne en Amérique du Nord, tant avant 2015 (fentanyl/ opioïdes extrêmement puissants : $1,8 \mathrm{mg}$ [intervalle interquartile $\{I I Q\}: 1,0-4,0]$; héroïne : $0,8 \mathrm{mg}$ [IIQ : 0,4-0,8]) qu'après 2015 (fentanyl/ opioïdes extrêmement puissants : 3,4 mg [IIQ : $3,0-4,1]$; héroïne : $2 \mathrm{mg}$ [IIQ : 1,4-2,0]). Dans les études où était indiquée la survenue d'événements indésirables, 11\% (490/ 4414) des sujets ont éprouvé des signes de manque.

La présentation des variables, l'hétérogénéité des études ainsi que leur piètre qualité limitent la portée de la conclusion. Conclusion: Les praticiens ont utilisé des doses initiales supérieures et, dans certains cas, des doses cumulatives supérieures de naloxone pour neutraliser la toxicité d'une exposition présumée au fentanyl ou à des opioïdes extrêmement puissants comparativement à d'autres opioïdes. Aussi faudrait-il mener des études comparatives de qualité sur les doses de naloxone afin d'en évaluer l'efficacité et l'innocuité.

Keywords: Toxicology, substance misuse, public health

\section{INTRODUCTION}

The ongoing opioid epidemic in North America has decreased life expectancy in the United States and Canada. ${ }^{1-8}$ National strategies aim to expand naloxone access to prevent opioid-related deaths. ${ }^{9,10}$ Since 2013 , fentanyl and ultra-potent opioids, $(\geq 50-100$ times more potent than morphine), are increasingly prevalent in North America. ${ }^{11-15}$ In 2015, the United States issued a nationwide alert on fentanyl, yet overdose deaths from fentanyl/ultra-potent opioids doubled from 20152016. ${ }^{11,16}$ British Columbia, the province at the epicenter of the Canadian epidemic, declared "fentanyl emergence" in the illicit drug supply in 2015 due to increased fentanyl detection in urine drug screens among people unaware of their fentanyl exposure. ${ }^{17,18}$ By 2018, fentanyl/ultra-potent opioids were detected in $85 \%$ of illicit overdoses in British Columbia. ${ }^{19}$

Naloxone is a competitive opioid antagonist that reverses opioid toxicity. ${ }^{20-22}$ However, it may have harmful effects in opioid-tolerant patients, if administered in higher doses than required. These include withdrawal (e.g., agitation, diaphoresis, vomiting) and other serious effects (e.g., pulmonary edema, seizures, dysrhythmias). ${ }^{23-30}$ The optimal dose likely differs by opioid type. ${ }^{24,31}$ Emerging evidence suggests that higher doses may be required in fentanyl/ultra-potent opioid toxicity. ${ }^{32,33}$ Despite an urgent need to update current guidelines to reflect changing epidemiology, the optimal naloxone dosing for fentanyl/ultra-potent opioid overdoses remains unknown. ${ }^{34,35}$ Existing reviews on naloxone dosing have not specifically examined data from presumed fentanyl/ultra-potent opioid overdoses, and, therefore, are not generalizable to jurisdictions with high fentanyl/ultra-potent opioid prevalence. ${ }^{22,36-38}$

Our main objective was to synthesize evidence on the relationship between initial naloxone dose and toxicity reversal among patients with undifferentiated and presumed fentanyl/ultra-potent opioid toxicity. Secondary objectives were to assess the relationship between cumulative naloxone dose and toxicity reversal, and between dose and adverse events.

\section{METHODS}

This systematic review was registered in PROSPERO (CRD42018096612), and follows Preferred Reporting Items for Systematic Reviews and Meta-Analyses guidelines. ${ }^{39}$ Details are available in a submitted protocol.

\section{Eligibility criteria}

We included studies examining people $>12$ years who received naloxone (any route, lay or health care responders) for toxicity from nonmedical opioid use. We chose 
this age cutoff because we hypothesized that adolescents respond similarly to adults. No comparator group was necessary. Included studies needed to document dose (initial and/or cumulative), and response and/or adverse events.

We included interventional and observational studies, and case reports/series to capture the most up-to-date evidence, given the novelty of fentanyl/ultra-potent opioid exposures, and the paucity of formal studies.

\section{Data sources and searches}

A professional librarian developed a primary search strategy in Embase using EMTREE subject headings and keywords from $>90$ relevant papers. We included all pertinent ultra-potent opioid indexing terms and synonyms. Our search included concepts naloxone AND drug overdose AND (adverse effects OR emergency care OR drug administration), and concepts naloxone AND (dosage OR administration OR adverse drug effects OR ultra-potent opioids). We searched for systematic reviews to October 3, 2018. We adapted subsequent searches to MEDLINE(Ovid), Cochrane Central Register of Controlled Trials, Database of Abstracts of Reviews of Effects, Cumulative Index to Nursing and Allied Health Literature, and Science Citation Index (Web of Science). We applied searches from 1972 to 2018 and limited to human studies with no language restrictions during title/abstract screening. Our full text review included relevant English citations, and French and German articles from Embase and MEDLINE(Ovid).

To identify grey literature, we searched Google using terms "naloxone," "opioid," "fentanyl," and "ultra-potent opioid." We searched websites and conference proceedings of professional toxicology organizations, harm reduction initiatives, opioid overdose guidelines, reference lists, and table of contents of prespecified journals.

\section{Study selection}

Two investigators assessed abstracts and full-text articles independently and in duplicate using a pilot-tested standardized form. We resolved disagreements through discussion or arbitration.

\section{Data extraction and quality assessment}

Two reviewers extracted data independently and in duplicate. Where reported, we extracted information on proportion of patients responding to specified doses. We attempted to email studies' authors twice for missing information.

Two independent investigators appraised English studies for risk of bias using an adapted Downs \& Black tool for observational and case studies/series ${ }^{40}$ and Cochrane Risk of Bias Tool for randomized controlled trials. ${ }^{41}$ One reviewer assessed French and German studies for inclusion, risk of bias, and extracted data.

\section{Definitions}

\section{Dosing}

We defined initial dose as the first naloxone dose administered. We stratified initial doses as $\leq 0.4 \mathrm{mg}$ (low) and $>0.4 \mathrm{mg}$ (high).

We defined cumulative dose as the sum of naloxone bolus doses. We excluded infusions, which are typically initiated after reversal to maintain ventilation and level of consciousness.

\section{Response}

We defined response to an initial dose if no additional doses were administered and patients remained alive. Due to inconsistent reporting, we were unable to specify a timeframe for response. We defined response to a cumulative dose if the patient remained alive after the overdose, regardless of additional treatments (e.g., infusions, intubation, other resuscitation).

\section{Data synthesis and analysis}

Due to high clinical/methodological heterogeneity, we did not meta-analyze results and instead synthesized them descriptively.

For each study, we abstracted total subjects, and number responding and/or experiencing an adverse event. If results were presented by dose received, we abstracted information for each dose level, and proportion responding. We performed subgroup analyses by confirmed or suspected opioid, study year, region, and location of administration (i.e., community, prehospital, or emergency department $<\mathrm{ED}>$ ).

For studies reporting response after presumed fentanyl/ultra-potent opioid toxicity, we plotted cumulative response probability against cumulative dose to identify variability in this association, and to assess whether we could recommend a uniform naloxone dose. 


\section{RESULTS}

\section{Study characteristics}

We identified 14,038 citations; 174 met inclusion criteria (Figure 1). There were 110 case reports/series, 57 observational studies, 6 randomized controlled trials, and 1 nonrandomized controlled trial. Publication dates ranged from 1972 to 2018, with 45 studies published after 2015, when North American sites started reporting increasing fentanyl/ultra-potent opioid prevalence. ${ }^{16,17}$ Included studies reported on 26,660 cases of opioid intoxication treated with naloxone. Geographically, 107 studies ( $\mathrm{n}=16,574)$ were completed in North America and 49 in Europe $(n=8,066$; Supplemental Online Appendix Table 1).

Median age was 35 years (interquartile interval [IQI], 25-41); 74\% were male. Forty studies reported heroin exposure, and 25 reported presumed fentanyl/ultrapotent opioid toxicity. Naloxone administration occurred in the community or prehospital in 55 studies, and in ED or hospital in 57 studies. Studies most commonly reported intravenous $(\mathrm{n}=119[60 \%])$, then intramuscular/subcutaneous $(n=48[24 \%])$, and intranasal $(n=24[12 \%])$ administration. Many reported multiple routes.

\section{Initial naloxone dosing}

Among studies reporting proportional response to initial naloxone dose, $60.3 \%(\mathrm{n}=48)$ responded in studies reporting doses $\leq 0.4 \mathrm{mg}$, and $70.1 \%(\mathrm{n}=66)$ in studies reporting initial doses $>0.4 \mathrm{mg}$. Some of these studies reported providing initial doses both $\leq 0.4 \mathrm{mg}$ and $>0.4$ $\mathrm{mg}$ to different groups. Where studies reported a low initial dose, $56.9 \%(617 / 1,085)$ of patients with presumed fentanyl/ultra-potent opioid exposure responded, v. $80.2 \%(170 / 212)$ of patients exposed to heroin. Where studies reported a high initial dose, 30.4\% (7/23) of patients with presumed fentanyl/ultra-potent opioid exposure responded, compared with 59.1\% (1,434/ 2,428 ) of patients exposed to heroin (Table 1). When we examined initial doses $\geq 1.2 \mathrm{mg}, 9.1 \%$ (1/11) and $56.4 \%(1,221 / 2,166)$ of patients with presumed fentanyl/ultra-potent opioid v. heroin exposure responded, respectively (Table 1). Among patients administered a higher initial dose, response rates were similar for different routes of administration. Initial doses administered increased over time. When studies were compared by publication year, fewer patients responded to low doses

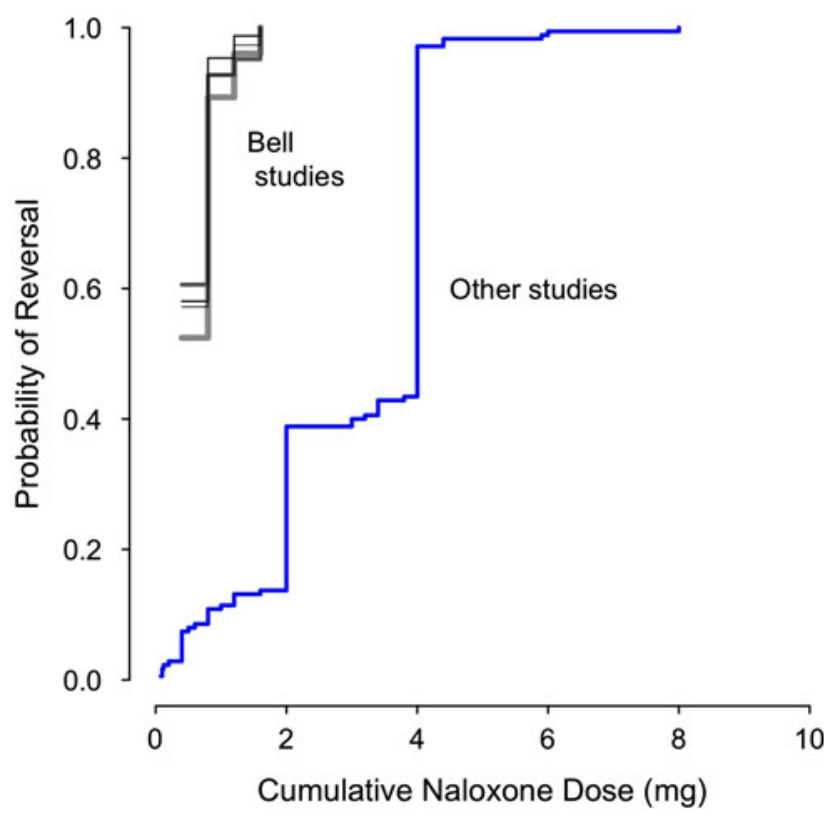

Figure 1. Evidence search and selection.

Table 1. Proportion of patient response to initial naloxone dose, by opioid exposure*

\begin{tabular}{|c|c|c|c|}
\hline Opioid exposure & $\begin{array}{l}\text { No. } \\
\text { ofstudies }\end{array}$ & $\begin{array}{l}\text { No. of } \\
\text { subjects }\end{array}$ & $\begin{array}{l}\% \\
\text { Response }\end{array}$ \\
\hline \multicolumn{4}{|c|}{ ultra-potent opioid } \\
\hline$\leq 0.4 \mathrm{mg}$ & 7 & 1085 & 56.9 \\
\hline$>0.4 \mathrm{mg}$ & 12 & 23 & 30.4 \\
\hline $0.4-1.2 \mathrm{mg}$ & 5 & 12 & 50.0 \\
\hline$\geq 1.2 \mathrm{mg}$ & 7 & 11 & 9.1 \\
\hline \multicolumn{4}{|l|}{ Heroin } \\
\hline$\leq 0.4 \mathrm{mg}$ & 8 & 212 & 80.2 \\
\hline$>0.4 \mathrm{mg}$ & 21 & 2428 & 59.1 \\
\hline $0.4-1.2 \mathrm{mg}$ & 8 & 262 & 81.2 \\
\hline$\geq 1.2 \mathrm{mg}$ & 13 & 2166 & 56.4 \\
\hline \multicolumn{4}{|l|}{ Other opioids } \\
\hline$\leq 0.4 \mathrm{mg}$ & 33 & 272 & 58.8 \\
\hline$>0.4 \mathrm{mg}$ & 33 & 3143 & 80.8 \\
\hline $0.4-1.2 \mathrm{mg}$ & 11 & 216 & 71.3 \\
\hline$\geq 1.2 \mathrm{mg}$ & 22 & 2927 & 81.5 \\
\hline
\end{tabular}

The boldfaced data are the main dosing categories. The non-boldfaced data $(0.4-1.2 \mathrm{mg}$, and $\geq 1.2 \mathrm{mg}$ ) refer to subcategories of the main category $>0.4 \mathrm{mg}$.

in more recent studies $(72.1 \%[\mathrm{n}=36]$ in studies before 2015 , v. $56.6 \%$ [ $\mathrm{n}=12$ ] in studies in 2015 and later).

Our subgroup analysis by location indicated lower responses to any initial dose administered in EDs 
(42.9\% [70/163]) v. community $(60.0 \%$ [945/1574]) or prehospital (68.7\% [2983/4343]).

\section{Cumulative naloxone dosing}

Seventy-five studies reported cumulative naloxone dose and proportion responding. Cumulative doses ranged from $0.04 \mathrm{mg}$ to $44 \mathrm{mg}$ among responders. Median cumulative dose among responders was higher for patients with presumed fentanyl/ultra-potent opioid exposure than heroin/other opioids in North America. Furthermore, median cumulative doses for all opioids increased in nearly all regions over time (Table 2).

By location, we found lower median cumulative doses in EDs $(0.4 \mathrm{mg}$ [IQI $0.08-1.6 \mathrm{mg}$ ]), v. community (0.8 mg [IQI $0.2-1.6 \mathrm{mg}$ ]) or prehospital (2.0 mg [IQI $2.0-2.0 \mathrm{mg}])$.

One epidemiological study ${ }^{42}$ described naloxone doses required to reverse overdoses in Pittsburgh from 2013 to 2016 ( $n=225,165,236,446$ per year), over which time there was increasing fentanyl prevalence in overdose deaths $(3.5 \%, 25.0 \%, 32.8 \%$, and $68.7 \%) .{ }^{42} \mathrm{We}$ generated annual dose-response curves using the 4 study years: a total dose of $0.8 \mathrm{mg}$ generated $89 \%$ reversal, and $1.6 \mathrm{mg}$ achieved $100 \%$ reversal. Annual dose-response curves were similar (Figure 2).

We combined data from remaining observational and case studies/series reporting presumed fentanyl/ultrapotent opioid toxicity in another dose-response curve (combined $n=182$ ). This indicated marked variability in cumulative dosing among patients responding, and higher overall doses than Bell ${ }^{22}$ : $0.8 \mathrm{mg}$ generated $11 \%$ reversal, and $4 \mathrm{mg}$ achieved $97 \%$ cumulative reversal (Figure 2).

\section{Adverse effects}

Adverse events reporting was inconsistent. Studies rarely specified events of interest a priori, and few reported dose received by individual patients experiencing adverse events. Therefore, we could not associate event occurrence with dose. Among 86 studies reporting adverse events, 41 described withdrawal, 17 pulmonary edema, and 11 seizures. It was often unclear if events were due to naloxone, or to the overdose. Among studies reporting adverse events, $11 \%(\mathrm{n}=490 / 4414)$ and $1 \%(\mathrm{n}=52 /$ 4414) of participants experienced withdrawal and pulmonary edema, respectively.

\section{Study quality}

Most studies were high risk of bias. Most did not define outcomes a priori (64\% no/undetermined) (Supplemental Online Appendix Figure 1). Of six randomized controlled trials, five were high and one was unclear risk of bias (Supplemental Online Appendix Figure 2).

\section{DISCUSSION}

\section{Interpretation of findings}

We aimed to synthesize evidence on naloxone dosing and reversal in undifferentiated and presumed fentanyl/ultra-potent opioid toxicity, given the urgent need to expand naloxone use to reduce opioid-related deaths. Our review suggests that lay and health care providers are using higher initial doses, and in some cases higher cumulative doses to reverse presumed fentanyl/

\begin{tabular}{|c|c|c|c|c|c|c|c|}
\hline \multirow[b]{2}{*}{ Drug type } & \multirow[b]{2}{*}{ Location } & \multicolumn{3}{|l|}{ Published from 1972-2014 } & \multicolumn{3}{|l|}{ Published in 2015 or later } \\
\hline & & No. of studies; subjects & Median & $|\mathrm{Q}|$ & No. of studies; subjects & Median & $|\mathrm{Q}|$ \\
\hline \multirow{3}{*}{$\begin{array}{l}\text { Presumed } \\
\text { fentanyl/ultra-potent opioid }\end{array}$} & North America & $7 ; 10$ & 1.8 & $1.0,4.0$ & $5 ; 12$ & 3.4 & $3.0,4.1$ \\
\hline & Europe & $1 ; 3$ & 0.35 & $0.3,0.6$ & $2 ; 10$ & 0.5 & $0.4,0.8$ \\
\hline & Other & $1 ; 1$ & 0.8 & $0.8,0.8$ & $0 ; 0$ & NA & NA, NA \\
\hline \multirow[t]{3}{*}{ Heroin } & North America & $10 ; 68$ & 0.8 & $0.4,0.8$ & $4 ; 18$ & 2 & $1.4,2.0$ \\
\hline & Europe & $1 ; 4$ & 0.4 & $0.4,0.7$ & $1 ; 250$ & 1.2 & $0.8,2.0$ \\
\hline & Other & $1 ; 148$ & 2.0 & $2.0,2.0$ & $0 ; 0$ & NA & $N A, N A$ \\
\hline \multirow[t]{3}{*}{ Other } & North America & $25 ; 56$ & 0.8 & $0.5,2.0$ & $7 ; 26$ & 0.1 & $0.07,2.0$ \\
\hline & Europe & $13 ; 51$ & 0.4 & $0.4,0.8$ & $1 ; 1$ & 0.8 & $0.8,0.8$ \\
\hline & Other & $0 ; 0$ & NA & $N A, N A$ & $1 ; 1$ & 0.2 & $0.2,0.2$ \\
\hline
\end{tabular}




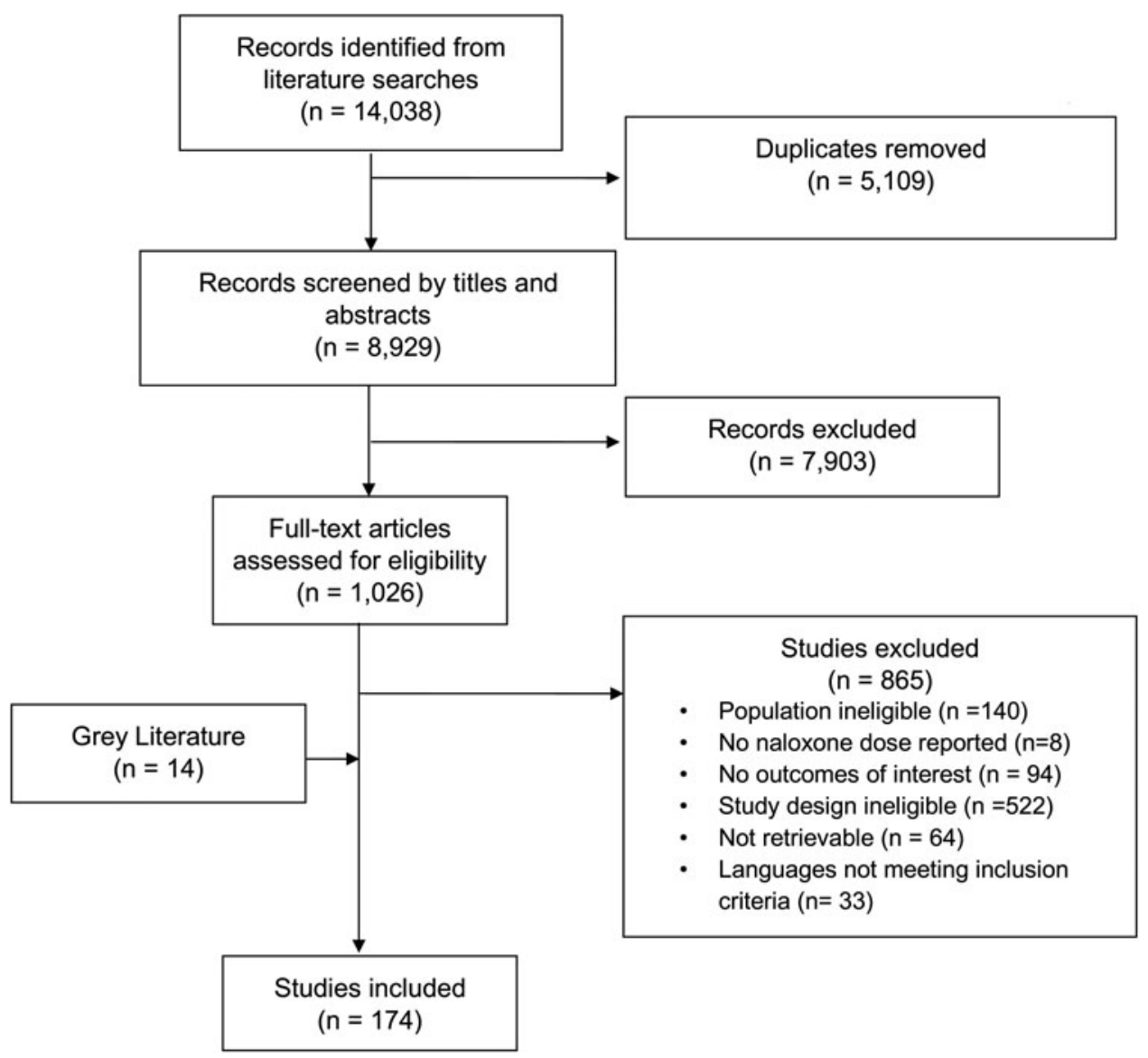

Figure 2. Cumulative dose-response curve for studies reporting presumed fentanyl/ultra-potent opioid exposures.

ultra-potent opioid toxicity, v. other opioids. Fewer patients responded to both low and high initial doses in presumed fentanyl/ultra-potent opioid overdoses v. heroin. In our analysis of cumulative dosing among patients responding, median doses for presumed fentanyl/ultra-potent opioid exposures were higher than for heroin/other opioids in North America, and increased over time. Our dose-response analysis indicated wide variability, and that a $4 \mathrm{mg}$ cumulative dose correlated with $97 \%$ reversal of presumed fentanyl/ ultra-potent opioid toxicity.

Importantly, we evaluated response among noncomparator studies. Therefore, response rates likely reflected differences among baseline populations (e.g., individual tolerance, co-morbidity), illness severity, or intoxicating opioid(s) (e.g., type, dose, route, potency). Lower response rates observed for higher v. lower initial doses in both presumed fentanyl/ultra-potent opioid and heroin exposures may be due to higher doses administered to patients with more severe toxicity (e.g., severe respiratory depression, or greater intoxicating dose). Similarly, lower responses to naloxone administered in EDs v. community/prehospital likely reflects that patients with more severe presentations are more likely transported to EDs. Our observations may also reflect provider behavior or clinical practice/protocols favoring higher doses over time and/or in North America. Alternatively, our findings may indicate that higher doses were required to reverse toxicity in settings with higher fentanyl/ultra-potent opioid prevalence (e.g., North America v. Europe/other regions). ${ }^{43,44}$

Our finding of lower median cumulative doses in ED v. community/prehospital settings may reflect different dosing protocols or administration routes (e.g., intranasal v. intravenous). Higher variability in cumulative doses in EDs likely indicates less protocol standardization compared with community/prehospital.

\section{Strengths and limitations}

This study is the most comprehensive systematic review to-date on naloxone dosing and the first to focus 
specifically on presumed fentanyl/ultra-potent opioid exposure. Compared with previous reviews, we have intentionally included nontraditional evidence sources (case reports/series) to capture recent fentanyl/ultrapotent opioid studies and ensure that our synthesis can inform urgently needed dosing protocols in areas with high fentanyl/ultra-potent opioid prevalence.

Our study is limited by inconsistent reporting. The specific rationale for initial and subsequent naloxone doses or routes were rarely provided. Therefore, we could not determine whether doses administered were necessary for reversal or driven by clinical practice/protocols. Parameters defining response were rarely outlined a priori, and timeframes were seldom clear. We therefore applied a simplified response definition (no additional doses, and patient remained alive) that we could uniformly apply. However, our definition may have misclassified patients who did not respond to a first dose, but whose ventilation was supported (e.g., intubation/noninvasive ventilation), who were administered additional dose(s) prematurely, or who initially responded, but required additional doses due to the intoxicating opioid's half-life.

Inconsistent reporting precluded us from adequately controlling for confounders (e.g., severity of toxicity) in our outcomes analysis. Additionally, our subgroup analyses of specific opioid exposures (e.g., fentanyl/ultra-potent opioids) relied on authors' classifications (suspected or confirmed). We likely missed or misclassified cases where the specific opioid type was unknown or unsuspected.

Our subgroup analysis based on location is limited because most studies reported multiple settings and, therefore, were not included. Furthermore, due to small denominators, we could not stratify results by presumed opioid exposure and specific dose administered.

Due to limited adverse events reporting, we were unable to summarize evidence regarding association of dose with adverse events. This prevents us from recommending dose and titration strategies that would maximize effectiveness while avoiding adverse events. Nonetheless, our finding that $11 \%$ of patients experienced withdrawal and $1 \%$ experienced pulmonary edema where reported indicates that these events are not rare and require specific consideration in future studies. Our findings align with existing reported rates of approximately $10-20 \%$ for minor events, and $1 \%$ for serious/life-threatening events. ${ }^{23,24,36}$

Finally, our systematic review is limited by overall poor study quality. We deliberately decided to include case studies/reports to ensure comprehensiveness. While this gives our study breadth, our results are limited by selection bias in these studies.

\section{Clinical and research implications}

Clinically, our results indicate that higher naloxone doses have been used in presumed fentanyl/ultra-potent opioid overdoses, suggesting a need to titrate naloxone to response. However, given the lack of high-quality comparative studies, we have not answered whether higher naloxone doses are effective and safe. Therefore, our results do not support changing current standard of practice, where health care providers administer an initial naloxone dose, concurrently support ventilation, and provide additional doses as needed. Our results have important implications in bystander settings where titrating naloxone to effect is limited by amount available to lay responders. Jurisdictions should carefully consider amount of naloxone available in take-home kits, although limitations of available evidence prevent us from recommending a specific dose. Current take-home naloxone kits in British Columbia contain three 0.4-mg doses. Instructions direct bystanders to provide ventilations, and administer additional doses in 3-5 minutes if no response (or sooner if rigidity). We recommend that other jurisdictions with increasing fentanyl/ultra-potent opioid prevalence consider similar approaches.

Our results should inform robust comparator studies evaluating different dosing strategies. High-quality prospective studies are needed to compare effectiveness and safety of low v. high initial and cumulative doses in this new fentanyl/ultra-potent opioid era. Future studies should analyze primary data from prehospital (ambulance), bystander, and ED settings to evaluate different dosing strategies. Additionally, our review highlights a need to standardize response definitions. Clearly timestamped vital signs and level of consciousness, before and after naloxone administration, would assist our understanding of clinical response to doses provided. Additionally, robust outcome measures would help to advance this field, by guiding future data collection, standardized registries, and allowing reliable analyses of pooled results in future systematic reviews.

\section{CONCLUSION}

In summary, in opioid toxicity due to presumed fentanyl/ ultra-potent opioid exposure v. other opioids, higher 
initial doses, and in some cases higher cumulative naloxone doses have been used by providers to achieve adequate reversal. Due to limitations of the available data, the relationship between naloxone dose and adverse events could not be answered.

Acknowledgments: The authors acknowledge the Centre for Clinical Epidemiology and Evaluation for providing space and in-kind support for this research.

Supplemental material: The supplemental material for this article can be found at https://doi.org/10.1017/cem.2019.471.

Financial support: This project was funded by a grant from the Canadian Institutes for Health Research (CIHR) for urgent Opioid Crisis Knowledge Synthesis (No. 397977), and a CIHR Foundation grant. Dr. Hohl's salary is supported by the Michael Smith Foundation for Health Research through the Health Professional Investigator Award.

Competing interests: None.

\section{REFERENCES}

1. Government of Canada. Measuring the impact of the opioid overdose epidemic on life expectancy at birth in Canada. 2018. Available at: https://www.canada.ca/en/health-canada/ services/substance-use/problematic-prescription-drug-use/ opioids/data-surveillance-research/harms-deaths/measuringimpact-on-life-expectancy.html (accessed January 24, 2019).

2. Ye X, Sutherland J, Henry B, Tyndall M, Kendall PRW. Impact of drug overdose-related deaths on life expectancy at birth in British Columbia. Health Promot Chronic Dis Prev Can 2018;38(6):248-51.

3. Special Advisory Committee on the Epidemic of Opioid Overdoses. National report: apparent opioid-related deaths in Canada (released March 2018). 2018. Available at: https:// www.canada.ca/en/public-health/services/publications/healthyliving/national-report-apparent-opioid-related-deaths-releasedmarch-2018.html (accessed January 24, 2019).

4. U.S. Department of Health \& Human Services. HHS Acting Secretary Declares Public Health Emergency to Address National Opioid Crisis. 2017. Available at: https://www. hhs.gov/about/news/2017/10/26/hhs-acting-secretarydeclares-public-health-emergency-address-national-opioidcrisis.html (accessed November 22, 2018).

5. Centers for Disease Control and Prevention. Drug Overdose Deaths; 2018. Available at: https://www.cdc.gov/drugoverdose/data/statedeaths.html (accessed January 24, 2019).

6. Murphy SL, Xu JQ, Kochanek KD, Arias E. Mortality in the United States, 2017. NCHS data brief, no 328. 2018 November 1-8.

7. Dowell D, Arias E, Kochanek K, et al. Contribution of opioid-involved poisoning to the change in life expectancy in the United States, 2000-2015. FAMA 2017;318 (11):1065-7.

8. Allen K. US life expectancy drops for 3rd year in a row, fueled by rising suicides, opioid deaths; ABC News November 292018.
9. U.S. Department of Health and Human Services. Strategy to combat opioid abuse, misuse, and overdose. Available at: https://www.hhs.gov/opioids/sites/default/files/2018-09/ opioid-fivepoint-strategy-20180917-508compliant.pdf (accessed November 18, 2019).

10. Government of Canada. Federal approach on Canada's opioid crisis. August 13, 2018. Available at: https://www.canada.ca/en/ health-canada/services/publications/healthy-living/federalapproach-canada-opioids-crisis.html (accessed November 27, 2019).

11. Centers for Disease Control and Prevention. Fentanyl. 2018. Available at: https://www.cdc.gov/drugoverdose/opioids/fentanyl.html (accessed February 11, 2019).

12. Armenian P, Vo KT, Barr-Walker J, Lynch KL. Fentanyl, fentanyl analogs and novel synthetic opioids: a comprehensive review. Neuropharmacology 2018;134(Pt A):121-32.

13. Centers for Disease Control and Prevention. Understanding the Epidemic. 2018. Available at: https://www.cdc.gov/drugoverdose/epidemic/index.html (accessed January 24, 2019).

14. Belzak L, Halverson J. Evidence synthesis - the opioid crisis in Canada: a national perspective. Health Promot Chronic Dis Prev Can 2018;38(6):224-33.

15. BC Coroners Service. Illicit drug overdose deaths in January 1, 2008 to November 30, 2018. BC Coroners Service December 27, 2018.

16. Drug Enforcement Administration. DEA issues nationwide alert on fentanyl as threat to health and public safety. 2015. Available at: https://www.dea.gov/press-releases/2015/03/ 18/dea-issues-nationwide-alert-fentanyl-threat-health-andpublic-safety (accessed November 18, 2019).

17. BC Centre for Disease Control. The BC public health opioid overdose emergency: March 2017. Update. 2017. Available at: http://www.bccdc.ca/resource-gallery/Documents/Educational\%20Materials/Epid/Other/Public\%20Surveillance \%20Report_2017_03_17.pdf (accessed August 7, 2018].

18. Amlani A, McKee G, Khamis N, Raghukumar G, Tsang E, Buxton JA. Why the FUSS (fentanyl urine screen study)? A cross-sectional survey to characterize an emerging threat to people who use drugs in British Columbia, Canada. Harm Reduct 7 2015;12(1):54.

19. BC Coroners Service. Fentanyl-detected illicit drug overdose deaths January 1, 2012 to September 30, 2018. December 27, 2018.

20. Trescot AM, Datta S, Lee M, Hansen H. Opioid pharmacology. Pain Physician 2008;11(2 Suppl):S153.

21. Ngai SH, Berkowitz BA, Yang JC, Hempstead J, Spector S. Pharmacokinetics of naloxone in rats and in man: basis for its potency and short duration of action. Anesthesiology 1976;44(5):398-401.

22. Boyer EW. Management of opioid analgesic overdose. $N$ Engl 7 Med 2012;367:146-55.

23. Buajordet I, Naess AC, Jacobsen D, Brors O. Adverse events after naloxone treatment of episodes of suspected acute opioid overdose. Eur 7 Emerg Med 2004;11(1):19-23.

24. van Dorp EL, Yassen A, Dahan A. Naloxone treatment in opioid addiction: the risks and benefits. Expert Opin Drug Saf 2007;6(2):125-32. 
25. Popper C, Kelen GD, Cunningham G. Naloxone hazard in drug abuser. Lancet 1989;2(8660):446.

26. Gaddis GM, Watson WA. Naloxone-associated patient violence: an overlooked toxicity? Ann Pharmacother 1992;26 (2):196-8.

27. Nath S, Tripathi M, Pandey C, Rao B. Naloxone-induced pulmonary edema: a potential cause of postoperative morbidity in laparoscopic donor nephrectomy. Indian $7 \mathrm{Med} \mathrm{Sci}$ 2009 Sep 25;63(2):72-5.

28. Michaelis LL, Hickey PR, Clark TA, Dixon WM. Ventricular irritability associated with the use of naloxone hydrochloride: two case reports and laboratory assessment of the effect of the drug on cardiac excitability. Ann Thorac Surg 1974;18 (6):608-14.

29. Cuss FM, Colaço CB, Baron JH. Cardiac arrest after reversal of effects of opiates with naloxone. Br Med 7 (Clin Res Ed) 1984;288(6414):363-4.

30. Andree RA. Sudden death following naloxone administration. Anesth Analg 1980;59(10):782-4.

31. Evans JM, Hogg MIJ, Lunn JN, Rosen M. Degree and duration of reversal by naloxone of effects of morphine in conscious subjects. Br Med 7 1974;2(5919):589-91.

32. Schumann H, Erickson T, Thompson TM, Zautcke JL, Denton JS. Fentanyl epidemic in Chicago, Illinois and surrounding Cook County. Clin Toxicol 2008;46(6):501-506.

33. Sutter ME, Gerona RR, Davis MT, et al. Fatal fentanyl: one pill can kill. Acad Emerg Med 2017;24(1):106-13.

34. Lavonas EJ, Drennan IR, Gabrielli A, et al. Part 10: special circumstances of resuscitation: 2015 American Heart
Association guidelines update for cardiopulmonary resuscitation and emergency cardiovascular care. Circulation 2015;132 (Suppl 2):S501-18.

35. Connors N, Nelson L. The evolution of recommended naloxone dosing for opioid overdose by medical specialty. 7 Med Toxicol 2016;12(3):276-81.

36. Chou R, Korthuis PT, McCarty D, et al. Management of suspected opioid overdose with naloxone in out-of-hospital settings: systematic review. Ann Intern Med 2017; 167(12):867.

37. Mueller SR, Walley AY, Calcaterra SL, Glanz JM, Binswanger IA. A review of opioid overdose prevention and naloxone prescribing: implications for translating community programming into clinical practice. Subst Abus 2015; $36(2): 240-53$.

38. Clarke SFJ, Dargan PI, Jones AL. Naloxone in opioid poisoning: walking the tightrope. Emerg Med $f$ 2005;22 (9):612-6.

39. Moher D, Liberati A, Tetzlaff J, Altman DG. Preferred reporting items for systematic reviews and meta-analyses: the PRISMA statement. BMF 2009;339(7716):332-6.

40. Downs SH, Black N. The feasibility of creating a checklist for the assessment of the methodological quality both of randomised and non-randomised studies of health care interventions. $\mathcal{F}$ Epidemiol Community Health 1998;52 (6):377-84.

References 41 to 215 are found in the Supplemental Online Appendix. 